\title{
The Coronavirus (Safeguards) Bill 2020 \\ Proposed protections for digital interventions and in relation to immunity certificates
}

\author{
Lead author: \\ Prof Lilian Edwards, University of Newcastle \\ Other contributors: \\ Dr Michael Veale, University College London \\ Dr Orla Lynskey, London School of Economics \\ Rachel Coldicutt, Careful Industries \\ Dr Nóra Ni Loideain, Institute of Advanced Legal \\ Studies, University of London \\ Frederike Kaltheuner, Mozilla Foundation \\ Marion Oswald, Northumbria University \\ Dr Rossana Ducato, UC Louvain \\ Prof Burkhard Schafer, University of Edinburgh \\ Prof Aileen McHarg University of Durham \\ Elizabeth Renieris, Harvard University \\ Elettra Bietti, Harvard University
}

\section{Version 5.1: 6 May 2020 \\ Feedback to lilian.edwards [at] ncl.ac.uk}

This Bill attempts to provide safeguards in relation to the symptom tracking and contact tracing apps that are currently being rolled out in the UK; and anticipates minimum safeguards that will be needed if we move on to a roll out of "immunity certificates" (commonly known as passports) in the near future. It does not mandate any particular technological approach to building apps nor does it attempt to duplicate the GDPR and ePrivacy Directive. Instead it suggests some basic safeguards that need to be placed on top of what these laws already supply.

Although no one wants to delay or deter the massive effort to fight coronavirus we are all involved in, there are clear reasons to put a law like this in place sooner rather than later:

(a) The portion of the population which is already digitally excluded needs reassurance that apps will not further entrench their exclusion

(b) Uptake of any contact tracing app, crucial to its success will be improved if the app is both trusted and trustworthy. Voluntary use is crucial to this. Accordingly we suggest a principle of non-compulsion which includes making sure that no-one such as employers or service providers can, as a way of compulsion, discriminate against those who have not installed or used the app.

(c) Connectedly, data quality will be much higher if people use these apps with confidence and do not provide false information to them, or withhold information, for fear of misuse or discrimination

(d) Both uptake and quality will also be improved if citizens feel they have rights to police these apps and the data gathered via them, via a robust and swift complaints mechanism

(e) Public trust will also be enhanced if an independent body whose remit is wider than just data protection has a watching brief to report on the whole scheme in relation to not just privacy but discrimination, freedom of movement, due process et al 
Accordingly this draft Bill makes it clear that

(a) No one shall be penalised for not having a phone (or other device), leaving house without a phone, failing to charge phone, etc

(b) No one is compelled to install a symptom and contact tracing app, or to share messages of their status on such an app (eg to an employer or insurer or university)

(c) Personal data collected by an app, or contained in an immunity certificate, shall not be shared beyond the NHS and coronavirus researchers unless securely anonymised.

(d) What is true, secure, verifiable, anonymisation needs to be certified by a stringent Code of Conduct

(e) Personal data collected by apps or immunity certificate must be deleted or anonymised as soon as possible, or at latest immediately after the emergency period has expired.

(f) "Immunity passports" must not become novel and uncontrolled internal passports, nor used by either state or private sector to discriminate in ways not necessary or proportionate to the legitimate social goal of controlling COVID-19.

\section{Bill contents:}

1. No sanctions for failing to carry personal device, install or run application

2. Data Protection Impact Assessment (DPIA): publicity requirement

3. No mandatory requirement to install application or display messages received by application without due safeguards

4. No repurposing or sharing of personal data derived from symptom tracking and contact tracing apps or processed in an immunity certificate

5. Deletion and anonymisation of data derived from symptom tracking, contact tracing apps and immunity certificates

6. Immunity certificates

7. Amendment to the Equality Act 2010

8. Review, complaints and oversight: Coronavirus Safeguarding Commissioner

10. Interpretation

10. Power to make delegated instruments under this Act

11. Commencement

12. Short title

13. Extent 


\section{A \\ B I L L}

TO

make provision for safeguards in relation to installation and collection, processing and sharing of data via symptom tracking and contact tracing apps; and for safeguards in relation to immunity certificates.

$\mathrm{B}$ E IT ENACTED by the Queen's most Excellent Majesty, by and with the advice and consent of the Lords Spiritual and Temporal, and Commons, in this present Parliament assembled, and by the authority of the same, as follows: -

\section{No sanctions for failing to carry personal device, install or run application}

(1) No person shall

(a) suffer, or be threatened with, criminal or civil sanctions or detention

(b) be denied, or be threatened with denial of, any public, contractual, employment or human rights, immigration status, benefits, immunities, opportunities or credits

solely because they

(i) do not own a personal device compatible with a symptom tracing app or contact tracing app;

(ii) fail to ensure at any time that their personal device is on their person or in a vehicle in which they are travelling, turned on, is in working order, has signal, has any protocol, such as Bluetooth, enabled, or is fully or partially charged.

\section{Data Protection Impact Assessment (DPIA): publicity requirement}

(1) Any Data Protection Impact Assessment required of any data controller under the General Data Protection Regulation (GDPR) art 35, in relation to either a symptom tracking or contact tracing app, or an immunity certificate, shall be made public for a consultation period of at least two weeks before the system is put into operation.

(2) Subs (2) shall apply mutatis mutandis whenever substantially new versions of any app or certificate are released.

\section{No mandatory requirement to install application or display messages received by application}

(1) No person shall 
(a) suffer, or be threatened with, criminal or civil sanctions or be subject to detention

(b) be denied, or be threatened with denial of, any public, contractual, employment or human rights, immigration status, benefits, immunities, opportunities or credits, or

(c) suffer discrimination under the Equality Act 2010

because they

(i) refuse or fail to install a symptom tracking or contract tracing app on a personal device

(ii) refuse or fail to display to any person messages sent to, or statuses disclosed by, such an app or

(iii) disable, delete or remove an app installed on their phone

unless an exception is laid down in Regulations by the Secretary of State.

(2) Negligently or innocently providing false or partial data to a symptom tracking or contact tracing app shall not constitute an offense under the Malicious Communications Act 1988 s 1(a)(iii).

(3) A parent or guardian shall be entitled to install a symptom tracking app or contact tracing app to the personal device of their child under 16, subject to (4) and (5) below

(4) A child shall have the right to veto such a choice or delete the app, if they are of sufficient age and maturity to understand the consequences of these actions

(5) Maturity in subs (3) above shall be rebuttably presumed at age 13.

\section{No repurposing or sharing of personal data derived from symptom tracking and contact tracing apps or processed in an immunity certificate}

(1) Personal data, including pseudonymous data, derived from, collected by or otherwise processed by a symptom tracking or contact tracing app, or processed by means of an immunity certificate, including but not restricted to the

(a) status of any person as an infected or potentially infected person;

(b) status of any person as a contact;

(c) location of any person

(d) proximity of any person

(e) status of any person as having previously been infected

(f) status of any person as immune and for what duration shall

(i) only be processed by the NHS or public health authorities, and

(ii) not shared to any other person for any purpose other than diagnosis, containment, treatment, research into or reduction of coronavirus disease

(iii) only be so shared after publication of a data sharing agreement and, if personal data is shared, a data protection impact assessment

(iv) If a DPIA is required, then there shall be prior consultation with the Information Commissioner under art 36 of the GDPR

(2) For the avoidance of doubt, any personal data processed by a symptom tracking or contact tracing app or immunity certificate shall be subject to the rules of the GDPR and 
the Data Protection Act 2018 including the rights, where appropriate, found in Ch III of the GDPR.

(3) In particular in relation to subs (2) above, a contact tracing app shall be regarded under art 22 of the GDPR as making a decision based solely on automated processing, including profiling, which produces legal effects or similarly significantly affects the owner of the personal device or any other persons whose personal data is processed.

(4) For the duration of the emergency period, for the purposes only of art 22(4) of the GDPR, processing of special category data on the lawful grounds of art 9(h) of the GDPR shall be deemed also to be lawful under art 9 (g) of the GDPR.

\section{Deletion and anonymisation of data derived from symptom tracking, contact tracing apps and immunity certificates}

(1) Personal data collected by or processed by symptom tracking or contact tracing apps or immunity certificates, must be deleted or anonymised as soon as the purpose of its processing is completed, or at latest after the end of the emergency period, by the data controller(s) or any other persons then processing it.

OR PHE]

.. at the latest at the end of 28 days [ or what period recommended as necessary by

(2) The Secretary of State shall indicate in a Code of Practice, after consultation with security and privacy experts, what steps, technical and organisational, must be taken to stringently anonymise personal data, or the purposes of subs (4) above.

(3) The Coronavirus Safeguarding Commissioner shall have the right to review before publication, and require amendments to, the Code in subs (5) above.

\section{Immunity certificates}

(1) No one except a police constable or, in England and Wales, a Community Support Officer, or a public sector prison warden, may require the display of an immunity certificate as a pre-condition for leaving the home, entering public spaces or using any form of private or public transportation.

(2) No person may require the display of an immunity certificate for any other purpose, or as a condition of any activity, except where such requirement

(a) is made publicly accessible

(b) any relevant data controller has issued an appropriate policy document under DPA 2018

(c) is in pursuit of the legitimate goal of diagnosis, containment, treatment, research into or reduction of coronavirus disease

(d) is necessary and proportionate to the goal in (c) above

(e) is limited in time to the minimum period necessary to achieve the goal in (c) above

(3) Any such requirement may be challenged by any person with interest to the relevant tribunal under s 8(8) below.

(4) Anyone denied an immunity certificate by a public authority may ask for such denial to be reviewed by the relevant tribunal under s $8(8)$ below. 


\section{Amendment to the Equality Act 2010}

Add to section 4 after "sexual orientation",

" coronavirus status as defined in s 8 of the Corona (Safeguards) Act 2020".

\section{Review, complaints and oversight: Coronavirus Safeguarding Commissioner}

(1) The operation of this Act shall be reviewed by a Coronavirus Safeguarding Commissioner ("the Commissioner") assisted by a number of Deputy Coronavirus Safeguarding Commissioners ("Deputies").

(2) The Prime Minister must appoint within three months of Royal Assent of this Act the Commissioner, and such number of Deputies as the Prime Minister considers necessary for the operation of this section.

(3) The terms of and qualifications for appointment of such Commissioner and Deputy Commissioners shall be made in regulations to be made by the Secretary of State.

(4) The Commissioner shall issue a report at least every month on the operation of this Act, in particular relating to its efficacy, any impact of the Coronavirus Act 2020 and Coronavirus Regulations on privacy or other human rights, and any discriminatory impacts of the above on sectors or classes of the population including but not restricted to persons with protected characteristics under the Equality Act 2010.

(5) Any report made by the Commissioner under (4) above shall be made public.

(6) The Commissioners shall consider within their reports under subs (4) whether any amendments to, additions to, or deletion of the safeguards contained in this Act should be recommended to Parliament, and if so, recommend such to the appropriate Minister who shall then convene a debate in Parliament.

(7) The Commission shall adopt a Technology Advisory Panel to provide advice to all Commissioners on the impact of any technology employed for any purposes referred to in this Act and to advise on the availability and development of such technologies while minimising interference with any rights protected under the Human Rights Act 1998.

(8) Any person who feels that their rights under this Act have been infringed may take a complaint to the First Tier Tribunal General Regulatory Chamber.

(9) Nothing in this section prevents any exercise of statutory duty by other public bodies, ombudsmen or regulators including the Information Commissioner's Office and the Equality and Human Rights Commission.

\section{Interpretation}

In this Act -

"coronavirus" and "coronavirus disease" have the meanings given in s 1(1) of the Coronavirus Act 2020

The "Coronavirus Regulations" are the Health Protection (Coronavirus) Regulations 2020 No 129, the Health Protection (Coronavirus) (Wales) Regulations 2020 No 308 (W. 68) and Health Protection (Coronavirus) (Restrictions) (Scotland) Regulations 2020 SSI No 103 and any other regulations made under the Coronavirus Act 2020 or under this Act 
A "symptom tracking app" is a computer program which can be installed on a user's personal device and which asks users to submit details of their symptoms, and optionally, other data, to a public health authority or their delegated agent, via electronic or other means

A "potentially infected person" (PIP) is defined as in the Coronavirus Act 2020, Sched 21, Part 1

A "contact incident" occurs where a PIP has come within 2 metres for 15 minutes or more of another living person (the "contact"), or what other definition is laid down from time to time by the Secretary of State

A “contact tracing app" is a computer program which can be installed on a user's personal device, and which -

(a) determines by a procedure to be designated in regulations by the Secretary of State that a contact incident has occurred

(b) takes consequent steps, such as

(i) reporting this contact incident to a public health authority or other public authority, whether straightaway or when a certain number of contact incidents has accumulated, or

(ii) providing guidance or instructions to the holder of the personal device or their household

A contact tracing app includes computer code that is installed within the operating system of the personal device of the user.

An "immunity certificate" is a certificate, paper or electronic, issued by a competent authority that inter alia a person has been infected with coronavirus in the past and is now presumed to be immune for a period of time and for defined purposes to be indicated in the certificate

A "personal device" includes a smartphone, tablet, laptop or desktop computer, wearable computer or beacon placed within or near a home.

"coronavirus status" is defined as "status as positive or negative for either being currently infected with, or having been infected with, coronavirus".

"personal data" is defined as in GDPR, art 4(1)

"processing" is defined as in GDPR, art 4(2)

"data controller" is defined as in GDPR art 4(7)

"consent" is defined as in GDPR, art 4(11)

“data protection impact assessment" is defined as in GDPR art 35.

“appropriate policy document" is defined as in DPA 2018, Sched 1.

"emergency period" is defined as in the Coronavirus Regulations 
Coronavirus (Safeguards) Bill 2020

10. Power to make delegated instruments under this Act

$$
[\ldots]
$$

\section{Commencement}

This Act comes into force on the day on which this Act is passed.

\section{Short title}

This Act may be cited as the Coronavirus (Safeguards) Act 2020.

\section{Extent}

$[\ldots]$ 